\title{
Development of an Anthropomorphic Breast Phantom for Combined PET, B-Mode Ultrasound and Elastographic Imaging
}

Jun Dang, Benjamin Frisch, Student Member, IEEE, Philippe Lasaygues, Member, IEEE, Dachun Zhang, Stefaan Tavernier, Member, IEEE, Nicolas Felix, Paul Lecoq, Member, IEEE, Etiennette Auffray, Joao Varela, Member, IEEE, Serge Mensah, Member, IEEE, and Mingxi Wan

\begin{abstract}
Combining the advantages of different imaging modalities leads to improved clinical results. For example, ultrasound provides good real-time structural information without any radiation and PET provides sensitive functional information. For the ongoing ClearPEM-Sonic project combining ultrasound and PET for breast imaging, we developed a dual-modality PET/Ultrasound (US) phantom. The phantom reproduces the acoustic and elastic properties of human breast tissue and allows labeling the different tissues in the phantom with different concentrations of FDG. The phantom was imaged with a whole-body PET/CT and with the Supersonic Imagine Aixplorer system. This system allows both B-mode US and shear wave elastographic imaging. US elastography is a new imaging method for displaying the tissue elasticity distribution. It was shown to be useful in breast imaging. We also tested the phantom with static elastography. A 6D magnetic positioning system allows fusing the images obtained with the two modalities. ClearPEM-Sonic is a project of the Crystal Clear Collaboration and the European Centre for Research on Medical Imaging (CERIMED).
\end{abstract}

Index Terms-Breast, elastography, PET, ultrasound.

\section{INTRODUCTION}

B REAST cancer is the second most common type of cancer in women [1] and the fifth most common cause of death worldwide [2]. X-ray mammography and B-mode ultrasound scanning are in routine clinical use today for breast cancer detection. However, the imaging methods from nuclear medicine, are only rarely used in the case of breast cancer.

Manuscript received February 23, 2010; revised May 26, 2010, August 23, 2010, and November 04, 2010; accepted January 03, 2011. Date of publication April 05, 2011; date of current version June 15, 2011.

J. Dang and S. Tavernier are with the Vrije Universiteit Brussel, 1050 Brussel, Belgium (e-mail: bmedangjun@gmail.com; stefaan.tavernier@vub.ac.be).

B. Frisch, P. Lecoq, and E. Auffray are with CERN, CH-1211 Geneve 23, Switzerland.

P. Lasaygues and S. Mensah are with the National Centre for French Scientific Research (CNRS)-LMA, 13402 Marseille Cedex 20, France (e-mail: lasaygues@1ma.cnrs-mrs.fr).

D. Zhang and M. Wan are with Xi' an Jiaotong University, Xi' an, Shaanxi 710049, China.

N. Felix is with SuperSonic Imagine, 13857 Aix en Provence, France.

J. Varela is with Laboratório de Instrumentação e Física Experimental de

Partículas (LIP), 1000-149 Lisboa, Portugal.

Color versions of one or more of the figures in this paper are available online at http://ieeexplore.ieee.org.

Digital Object Identifier 10.1109/TNS.2011.2105279
X-ray mammography has a sensitivity of about $90 \%$ for the detection of breast cancer [3], but its specificity for distinguishing malignant from benign lesions is only about $20-50 \%$ (i.e., there are many false positives) [4]. Thus, a large fraction $(>50 \%)$ of the suspicious structures identified is benign, and further investigation is necessary before deciding to treat the patient for cancer [5]. This is to be compared with the sensitivity and specificity of PET for detecting breast cancer. These are reported to be $75 \%$ to $100 \%$ and $79 \%$ to $97 \%$, respectively [6]-[9], which is ideal for the detection of malignant tumours.

On the other hand, ultrasound (US) is a commonly used imaging modality for breast cancer imaging. It has a diagnostic sensitivity of $80 \%-100 \%$ and a specificity of $64 \%-99 \%$ [10]-[12] for breast cancer, as well as excellent temporal and spatial resolution [13]. It provides anatomical information and can even provide functional information if used with US contrast agents. In addition, US is a low-cost, real-time imaging technology that delivers no radiation dose to the patients.

US elastographic imaging devices, in addition to providing morphological information by US B-mode imaging, also provide the tissue's elasticity distribution. The application of this technology to breast imaging was shown to give promising results [14]. It will provide not only the functional and morphological information, but also the tissue elasticity distribution in the breast.

Combined PET/CT scanners are now in routine clinical use. However, CT provides poor contrast in soft tissues such as in breast and the prostate [15]; therefore, PET/CT is not well suited for breast multimodal imaging. On the contrary, US imaging provides good soft-tissue contrast.

All the above considerations motivated the members of the ClearPEM-Sonic collaboration to develop the combination of PET with US elastography. Dual PET/US breast imaging could possibly be useful to guide biopsy, to guide treatment procedures, and could allow detecting cancer at an earlier stage than it is currently possible.

ClearPEM is a dedicated PET scanner for breast imaging developed by the Crystal Clear Collaboration and produced by PETSys (Lisbon, Portugal). In the name "ClearPEM", PEM stands for Positron Emission Mammography. The machine was shown to have excellent performance [16]. The ClearPEM-Sonic dual-modality PET/US scanner is a combination of a ClearPEM breast imager and an Aixplorer US system produced by Supersonic Imagine, into a dual imaging device 
allowing to acquire PET and US images during the same patient imaging session. The breast will be immobilized by a plastic breast contention cone tailor made to the shape and volume of the breast. The ultrasound probe is held by an arm and will be manually pressed against the breast, using a window for US probe in the breast contention cone.

Ultrasound phantoms are commercially available, but there are no commercially available phantoms for combined PET/US. The main motivation for the present project was developing a phantom that can be used with the ClearPEM-Sonic dual-modal PET/US scanner. Different parts in the phantom should have acoustic properties that are typical for human breast tissues and, at the same time, should be impregnated with positron emitting tracers at concentrations that are typical of what is present in human breast during PET scans. In addition, the phantom should also reproduce the elasticity of real tissues in the breast so that the phantom can also be used for US elastographic imaging. In the test reported here the phantom was evaluated separately with a standard whole-body PET system and with the Aixplorer elastographic US system. Moreover, we also tested the phantom with static elastography, which is another method for elastography imaging.

\section{MATERIALS AND MethodS}

The phantom was made of mixed gels of gelatine (GELITA EUROPE, Ballistic 2, photographic grade) and "high gel Agar" (SIGMA-ALDRICH Co.). However, such gels will degrade within a few days after production. We found that adding less than $0.5 \%$ of Germall-plus [15] as a conservation agent greatly improved the conservation properties of the gel, and in this way, the samples were stored for several weeks in a fridge. To find gels with the desired properties, we measured the velocity and the attenuation of the acoustic waves and Young's modulus (the modulus of elasticity) of a large number of samples. The acoustic velocity and the attenuation coefficient were measured in a set-up with two parallel single US transducers immersed in a water tank, and with the sample between the transducers [17]. Young's modulus was measured by static stretching. The stretching force and corresponding deformation were recorded, and the slope gives the sample's Young modulus.

We evaluated samples with gelatine mass percentages varying from $1 \%$ to $15 \%$ and with agar mass percentages varying from $0.5 \%$ to $6 \%$. Mixed gels with more agar than gelatine remained liquid and could not be used for making phantoms. We found that the acoustical velocity of the waves mainly depends on, and increases with, the gelatine fraction, and that the attenuation of the waves mainly depends on, and increases with, the agar fraction. This allowed us to find gel compositions that gave samples with acoustic and elastic signature similar to fat tissue, normal glandular tissue, fibrous/hard tissue, and carcinoma in the breast. Table I lists a few of the gels tested and their acoustical properties.

Table II shows the acoustic properties and Young's moduli for real breast tissues reported in the literature [18], [19]. A comparison of Tables I and II shows that it is possible to find gels that closely mimic the properties of real breast tissues.

We chose a gel with $1 \%$ gelatine and $0.5 \%$ agar to mimic the fat medium in the breast and a gel with $12 \%$ gelatine and $4 \%$
TABLE I

ACoustical Properties and Young's Moduli for Mixed Gelatine-Agar SAMPLES With a SMALl AMOUNT OF GERMALl-Plus

\begin{tabular}{c|c|c|c|c}
\hline \multicolumn{2}{c|}{ Composition } & \multirow{2}{*}{$\begin{array}{c}\text { Acoustical } \\
\text { wave velocity } \\
{[\mathrm{m} / \mathrm{s}]}\end{array}$} & $\begin{array}{c}\text { Acoustical } \\
\text { wave } \\
\text { attenuation } \\
{[\mathrm{db} / \mathrm{Hz} / \mathrm{mm}]}\end{array}$ & $\begin{array}{c}\text { Young's } \\
\text { modulus } \\
{[\mathrm{KPa}]}\end{array}$ \\
\hline $1 \%$ & $0.5 \%$ & $1503 \pm 8$ & $0.05 \pm 0.02$ & $22 \pm 2$ \\
$5 \%$ & $2 \%$ & $1510 \pm 4$ & $0.10 \pm 0.02$ & $33 \pm 1$ \\
$12 \%$ & $4 \%$ & $1543 \pm 11$ & $0.13 \pm 0.02$ & $73 \pm 9$ \\
$15 \%$ & $6 \%$ & $1550 \pm 7$ & $0.25 \pm 0.02$ & $117 \pm 8$ \\
\hline
\end{tabular}

TABLE II

ACOUSTICAL PROPERTIES AND Youngs's MOdULI FOR REAL BREAST TisSUES

\begin{tabular}{c|c|c|c}
\hline Real tissue type & $\begin{array}{c}\text { Acoustical } \\
\text { wave } \\
\text { velocity[m/s] }\end{array}$ & $\begin{array}{c}\text { Acoustical } \\
\text { attenuation } \\
{[\mathrm{dB} / \mathrm{Hz} / \mathrm{mm}]}\end{array}$ & $\begin{array}{c}\text { Young's } \\
\text { modulus } \\
{[\mathrm{kPa}]}\end{array}$ \\
\hline $\begin{array}{c}\text { Fat } \\
\text { Glandular/Fibrous } \\
\text { tissue }\end{array}$ & $1479 \pm 32$ & 0.05 & $19 \pm 7$ \\
Carcinoma & $1553 \pm 35$ & 0.14 & $33 \pm 11$ \\
\hline \multicolumn{2}{|c}{} \\
\hline
\end{tabular}

agar to mimic hard tissue and carcinoma. All phantoms consist of a volume of gel simulating fat tissue with a number of inserts of varying dimensions (diameters from 1 to $3 \mathrm{~cm}$ ), simulating cancer lesions. For the positron emitting tracer concentration we used $4 \mathrm{kBq} / \mathrm{ml}$ in the fat tissue and $16 \mathrm{kBq} / \mathrm{ml}$ in the cancer lesions.

The phantom was prepared as follows:

1) To make gels simulating cancer lesions, $12 \%$ gelatine and $4 \%$ agar were mixed with the correct amount of deionized water, and heated in the microwave oven until the liquid nearly boils. The mixture was stirred moderately and cooled to $45^{\circ} \mathrm{C}$, and FDG was added as needed. Moulds were used to make "tumour volumes" of desired size and shape, and were solidified in a fridge.

2) To make gels mimicking fat tissue, $1 \%$ gelatine and $0.5 \%$ agar were mixed with the correct amount of deioniszed water, and the beaker with the mixture in a microwave oven was heated until the temperature reaches $70^{\circ} \mathrm{C}$ till all the gelatine and agar dissolved. The mixture was cooled down to $40^{\circ} \mathrm{C}$ and FDG was added as needed.

3) The "tumour volumes" were removed from the molds and inserted into the phantom while the gel simulating fat tissue was still at $40^{\circ} \mathrm{C}$.

4) The whole phantom was left to cool to room temperature where it solidified.

The time taken for preparing the phantom, including the cooling phase, was about $1 \mathrm{~h}$. The PET scan should be taken immediately after the preparation of the phantom, i.e., before the tracer decays. The US scan can be taken any time after that.

\section{RESULTS}

\section{A. Description of the Equipment}

The PET images of the phantoms were obtained with a PHILIPS GEMINI TF 64 scanner. We used a modified 
DP-9900 scanner (Mindray, P. R. China) for studying static elastography, and a SuperSonic Imagine Aixplorer scanner (SuperSonicImagine, Aix en Provence, France) for obtaining shear wave elastographic images.

The static elastogram obtained with the modified DP-9900 scanner is a strain image and not an image of Young's modulus. The Aixplorer scanner produces real elastograms, i.e., images of Young's modulus. This machine allows obtaining elastograms in a way that is independent of the amount of compression applied by the operator and therefore gives more reproducible results. SuperSonic Imagine uses a proprietary concept to introduce the shear wave into the body: ultrasound beams are focalized successively at different depths in the tissue, creating a shear wave in the medium [14], [20]. Using ultrafast imaging, the velocity of the shear wave at every point in the image is measured, and a quantitative elasticity map can be obtained. The result is a real-time, quantifiable, user-independent, and reproducible ShearWave Elastographic image.

\section{B. Tests With a Cuboi-Shaped Phantom}

In the first experiment, we studied static elastography with a rectangular cuboid-shaped phantom measuring $134 \mathrm{~mm} \times$ $88 \mathrm{~mm} \times 70 \mathrm{~mm}$. The phantom contained several globular inclusions representing cancerous lesions measuring between 10 and $40 \mathrm{~mm}$, immersed in a homogeneous volume of fat tissue mimicking medium.

In this study, a linear US probe with nominal frequency of $7.5 \mathrm{MHz}$ and sampling frequency of $25 \mathrm{MHz}$ was used. A rectangular compressor with a polyethylene plane of size $88 \mathrm{~mm}$ $\times 66 \mathrm{~mm}$ was used to enlarge the US probe's contact surface. The US probe was inserted in a slot cut in the compressor plane. US radio frequency (RF) data were acquired with a 32 bit data I/O card. Both pre- and post-compression US RF data were acquired. The images obtained with this setup, the compression plate, and typical signals are shown in Fig. 1.

For this measurement a compression of $0.5 \mathrm{~mm}$ was used. The strain image was derived from the difference in the RF data before and after compression. The local tissue displacements were estimated using the 1D cross-correlation technique by comparing the gated pre- and post- compression RF signals [21]. For all the static elastograms, the length of the correlation window was fixed at $2.5 \mathrm{~mm}$, and an overlap window of $80 \%$ was used. To improve the correlation between the pre- and postcompression signals, and therefore to improve the measurement of the displacements, temporal stretching [22] is applied to the post-compression signals with a stretching factor equal to the mean strain. After obtaining the displacement data, the axial strain was computed from the displacement estimates using a third-order optimum low-pass differentiator [23]. The dynamic range displayed in the strain image is $1.5 \%$.

Fig. 2 shows the images obtained with different modalities for the cuboid-shaped phantom with the size of $134 \mathrm{~mm} \times$ $88 \mathrm{~mm} \times 70 \mathrm{~mm}$. The white rectangle in the PET image shows the US scan region. In B-mode imaging, the tumour shows as a brighter region, which means that the tumour reflects the sound waves more strongly. In static elastography, the tumour shows

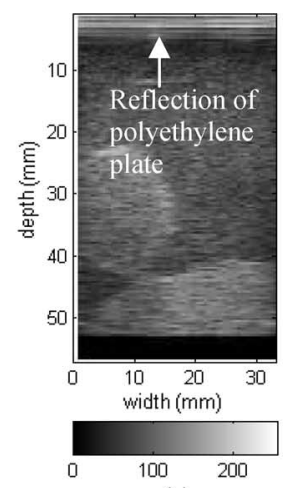

(a)

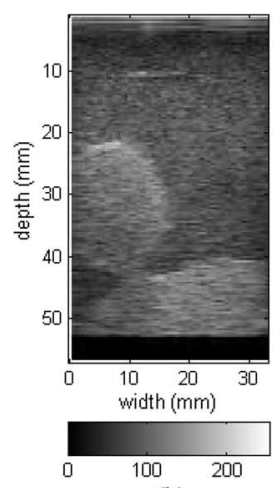

(b)

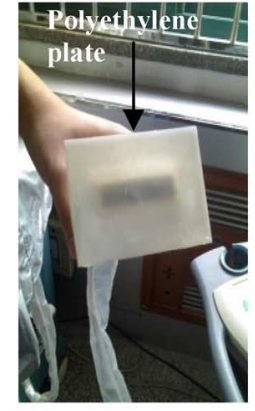

(c)

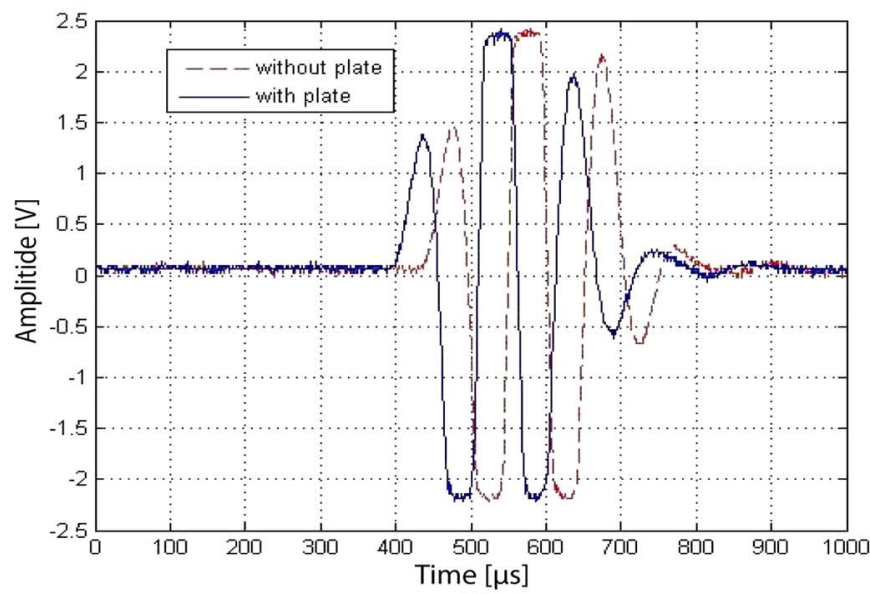

(d)

Fig. 1. Comparison of results obtained with and without polyethylene plate compressor, and the corresponding 1D ultrasound radiofrequency signals. (a) B-mode image of the phantom with the US probe fixed inside the polyethylene compressor plate, (b) B-mode image obtained without the polyethylene compressor plate, (c) US probe with its polyethylene compressor, and (d) comparison of the RF signals with and without the polyethylene compressor plate. The attenuation coefficient of the polyethylene plate had been tested to be very near to $0 \mathrm{~dB} / \mathrm{mm}$, so we believe it can be seen transparent to the US RF signals.

up as a darker region, which means that the strain distribution in the hard tumour parts is much smaller than in the softer fat-simulating material. The phantom was scanned for $2 \mathrm{~min}$ in a PHILIPS GEMINI TF 64 scanner, resulting in a total of $3.6 \times 10^{6}$ counts. The default energy window of $440-665 \mathrm{keV}$ was used. The PET data were acquired in list mode, and a TOF (time-of-flight) list-mode OSEM algorithm was used for image reconstruction. The OSEM algorithm has 33 subsets and stops after 3 iterations [24], [25]. Attenuation correction is performed using the CT transmission data, whereas scatter is estimated using a single-scatter simulation algorithm [24].

\section{Image Superposition}

Fig. 3 shows images of another cuboid shaped phantom $(134 \mathrm{~mm} \times 88 \mathrm{~mm} \times 60 \mathrm{~mm})$ obtained with the SuperSonic Imagine's Aixplorer shear wave elastographic imager. This figure shows the images obtained with US B-mode imaging, with ShearWave elastograpy, and with a whole-body PET. The superposition is also shown. The edges of the cuboid-shaped phantom are clearly visible on the PET image. Therefore, with this phantom, the relative position of the two images could simply be obtained with a ruler measuring the position of the 


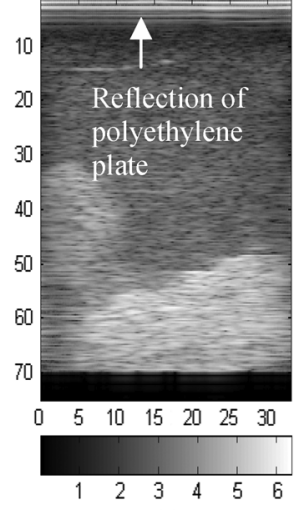

(a)

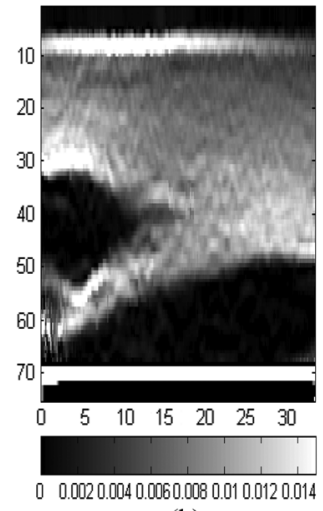

(b)

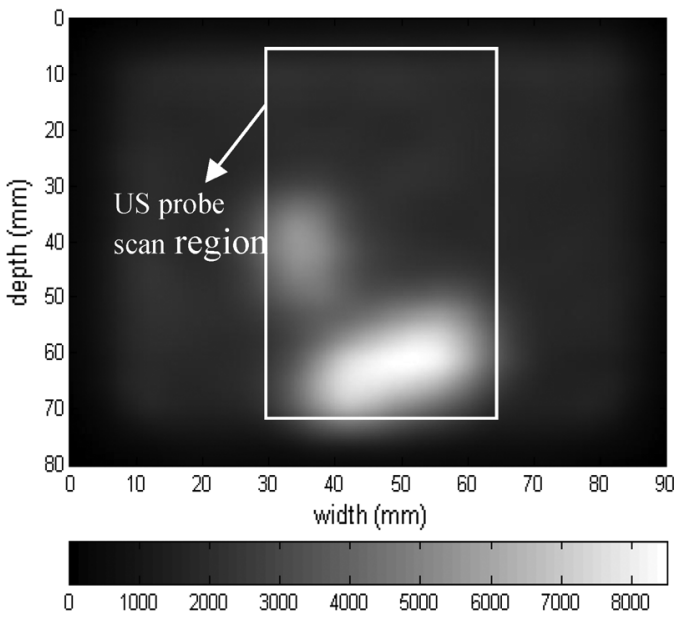

(c)

Fig. 2. Corresponding planes in the images obtained with different modalities for the cuboid shaped phantom. (a) B-mode US image $(506 \times 239$ pixels, field of view $69 \mathrm{~mm} \times 33 \mathrm{~mm})$, (b) static elastogram $(506 \times 239$ pixels, field of view $69 \mathrm{~mm} \times 33 \mathrm{~mm}$ ), and (c) PET image(interpolated as $540 \times 660$ pixels to have the same voxel size as US images, field of view $90 \mathrm{~mm} \times 80 \mathrm{~mm}$ ). The white box in the PET image shows the US probe scan region. The dynamic range of $\mathrm{B}$-mode image is $65 \mathrm{~dB}$. For elastogram the strain dynamic range is 0 to 0.015 .

US probe relative to the edges of the cuboid and by making sure the US probe was held vertically.

For clinical images taken with ClearPEM-Sonic, this simple approach will not be possible. In this case we will rely on a 6D magnetic positioning system (3D Guidance trakSTAR, Ascension Tech. Co.). According to the specifications, the spatial accuracy of the tracking system is $1.4 \mathrm{~mm} \mathrm{r.m.s} \mathrm{for} \mathrm{the} \mathrm{po-}$ sition and 0.5 degree r.m.s for the orientation angles. In the ClearPEM-Sonic system, the breast will be contained in a plastic holder that is well adapted to the shape and size of the breast. If this breast container has fiducial markers that are visible in the PET image and if the positions of these fiducials can be measured by the magnetic tracking system, it is possible to superimpose both images.

\section{Tests With the Anthropomorphic Phantom}

To test this idea we made an anthropomorphic breast phantom by filling one of the breast contention cones with breast-simulating gelatine-agar gels (Fig. 4(a)). For this phantom, as for the orthogonal cuboid-shaped phantom, the bulk of the breast tissue was taken to be fat, and a number of cancerous lesions of

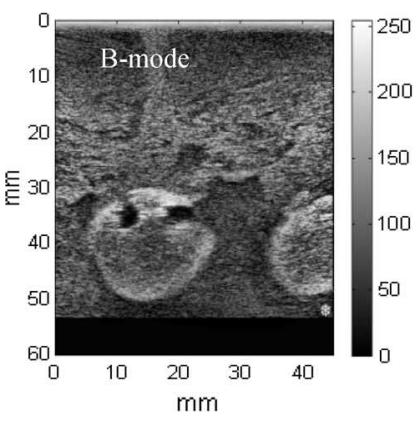

(a)

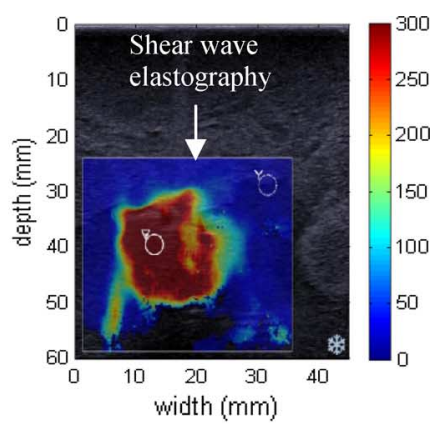

(b)

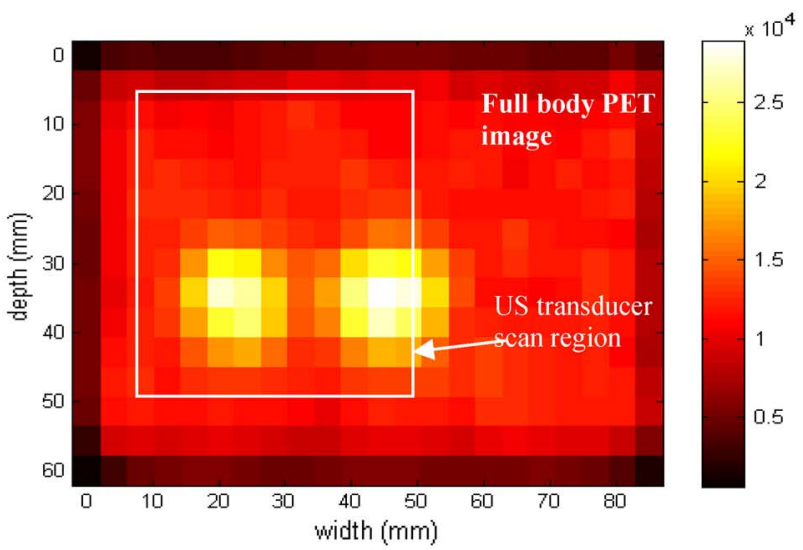

(c)

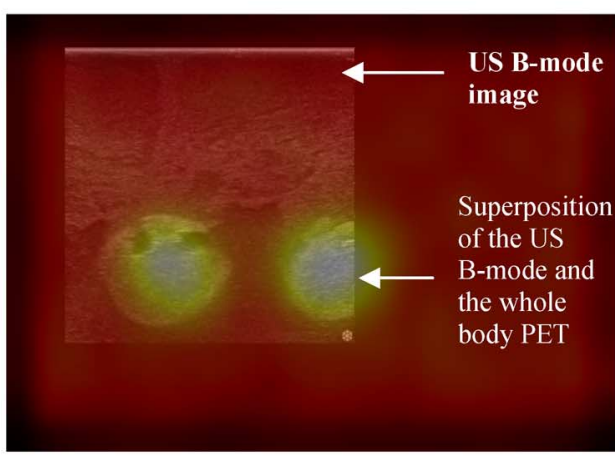

(d)

Fig. 3. Images of the cuboid-shaped phantom obtained with Supersonic Imagine's Aixplorer scanner and with a whole body PET: (a) B-mode image (749 $\times 711$ pixels, field of view $60 \mathrm{~mm} \times 45 \mathrm{~mm})$, (b) shear wave elastography $(749 \times 711$ pixels, field of view $60 \mathrm{~mm} \times 45 \mathrm{~mm})$, (c) whole-body PET image $(15 \times 22$ pixels, field of view $60 \mathrm{~mm} \times 88 \mathrm{~mm})$, white box stands for the US probe scan region, and (d) superposition of the two images (interpolated as $1050 \times 1573$ pixels, field of view $60 \mathrm{~mm} \times 88 \mathrm{~mm}$ ). Young's modulus ratio between tumour and tissue background is $6: 1$. The dynamic range of B-mode images is $65 \mathrm{~dB}$ and the elasticity range of elastography information is $300 \mathrm{kPa}$.

various sizes (diameters $1-2 \mathrm{~cm}$ ) were inserted in the volume. As fiducial markers, we used three low-activity (few micro-Ci) point sources. The plastic breast container cone, with the three fiducial markers attached to it, is shown in Fig. 4(a). The breast contention cone has a window for placing the US probe. For our test this window was covered with a Mylar foil. The width of the window is the same as the width of the US 3D probe width.

In the present test, we used a whole-body PET/CT scanner for obtaining the PET images. We found that the activity in the fiducial markers was too low for these to be clearly visible in the PET image. Fortunately, with a whole-body PET/CT scanner, the field of view is very large and the 10 metal screws close to 


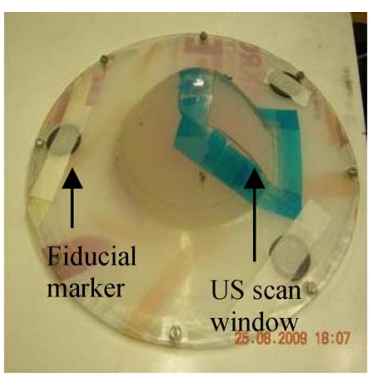

(a)

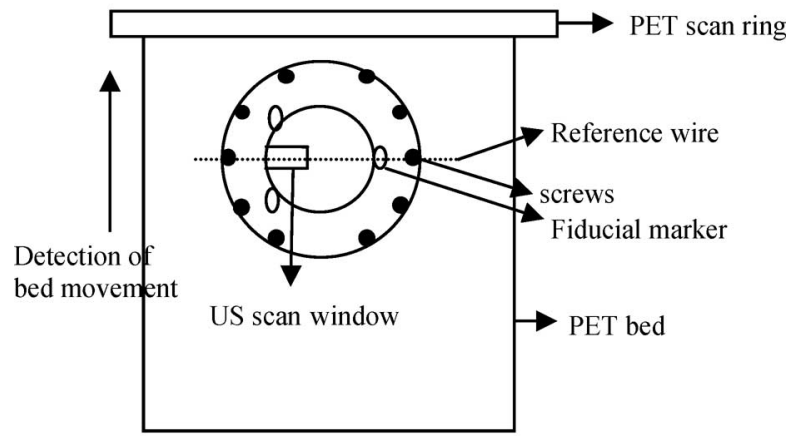

(b)

Fig. 4. (a) Breast shaped phantom and in a plastic breast contention cone. The positioning fiducials are the three gray discs, $20 \mathrm{~mm}$ in diameter. (b) Positioning of the phantom on the bed of the PET/CT scanner.

the edge of the phantom are in the field of view. We therefore relied on these metal screws instead. The diameter of the screws is $5 \mathrm{~mm}$. These are clearly visible in the CT image, and their position can also be measured with the magnetic positioning tracker. When performing the test, the phantom was also equipped with a wire strung horizontally in the symmetry plane of the breast contention cone. See Fig. 4(b). With the help of the laser beams, we could therefore position the phantom horizontally and exactly in the middle of the PET/CT image with the wire, and therefore the symmetry plane of the phantom, perpendicular to the axis of the scanner.

The US probe has a smooth and rounded shape so that it is comfortable in the operator's hand, but it has no suitable points for attaching the magnetic positioning probes. We therefore made a small plastic piece that can be attached to the US probe and that is equipped with two groves housing two magnetic probes in such a way that the axis of the US probe is exactly in the middle of, and exactly parallel to, the axes of the two magnetic positioning probes (Fig. 5(a)). The distance between the tip of the magnetic probes and the front of the US probe was measured with a ruler and found to be $80 \mathrm{~mm}$.

Using the information provided by the positioning probes, we made sure that while recording the US image, the US probe was held such that its central image plane coincided with the symmetry plane of the breast phantom. During the US scan, we recorded the position and the dip angle of the US probe. In this way, the central plane of the US image coincides with the symmetry plane of the breast phantom, and this symmetry plane coincides with the transaxial plane in the center of the PET image. The metal screw allows us to check the correct axial location of the phantom in the PET scanner. Finally we only need to apply the correct magnification factor and rotate the US

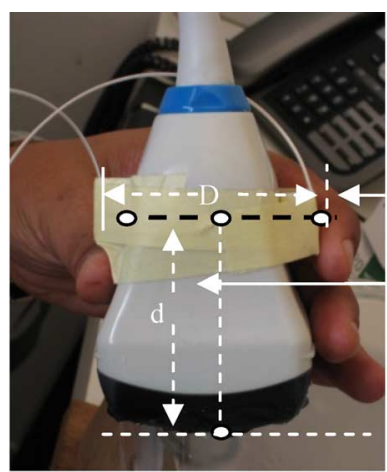

Magnetic probe holder. $\mathrm{D}=46 \mathrm{~mm}$

Distance $(\mathrm{d}=80$ $\mathrm{mm}$ ) from image region to the sensors

(a)

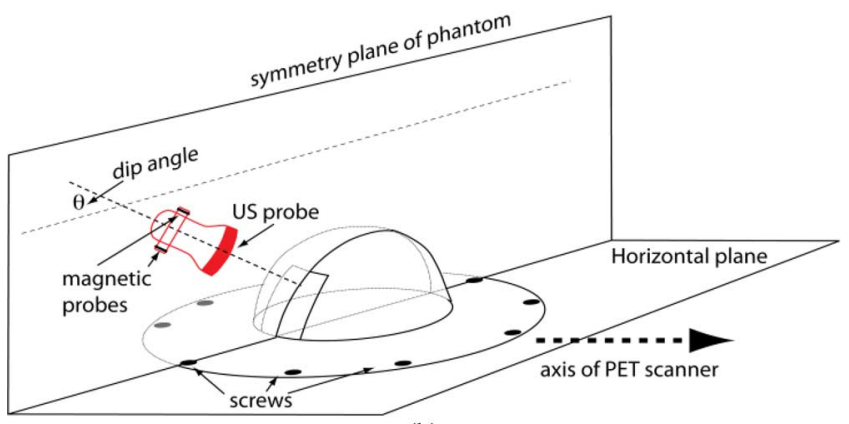

(b)

Fig. 5. US probe with positioning tracker and US/PET image co-registration protocol.

image by the measured dip angle to superpose both images, see Fig. 5(b).

Several factors influence the accuracy of the image fusion: First, the US probe deforms the breast when it is making contact. The deformation of the breast is several millimeters at the point of maximum deformation. The uncertainty of the position of the probe is $1.4 \mathrm{~mm}$ r.m.s. This has to be multiplied by $\sqrt{2}$ because both the fiducial and the probe position are measured. The uncertainty on the orientation of the probe translates into an uncertainty of $0.8 \mathrm{~mm}$ r.m.s on the position of the elevation of the US probe. Finally the PET image has voxels of $4 \mathrm{~mm}$. Considering all these factors, it is very likely that the relative position error of the PET and US is at most on PET voxel.

Fig. 6 shows the work flow and 2D fusion result in the central transaxial plane of the PET image.

\section{Discussion AND CONCLUSION}

\section{A. Comparison Between B-Mode US Image With Static Elastography}

The results presented here confirm the conclusions of several previous studies [26]-[28] regarding the good performance of static elastography compared to B-mode US imaging.

Fig. 7 illustrated the differences. This figure shows corresponding slices through the US B-mode image and the elastographic images obtained with the cuboid-shaped phantom. The top arrow in Fig. 7(b) points to a bright, high-strain region between two hard tumours. This seems to indicate the presence of very soft material, while in fact it is a mechanical artifact [26], [29] when compared with Fig. 7(a). In Fig. 7(c) and 7(d), the white arrow points at a small lesion that is hardly visible 


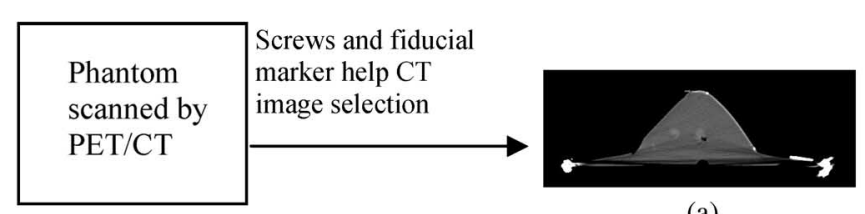

(a)

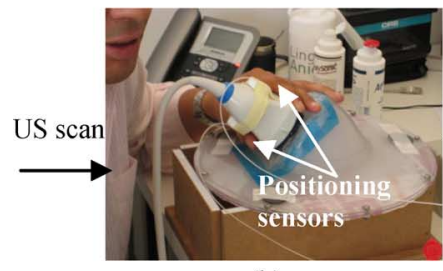

(b)

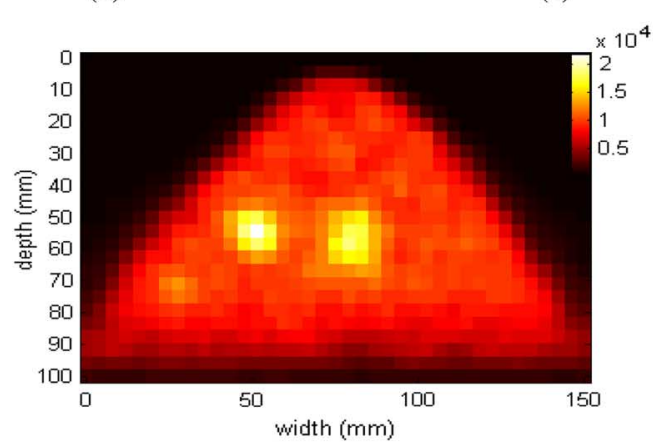

(d)

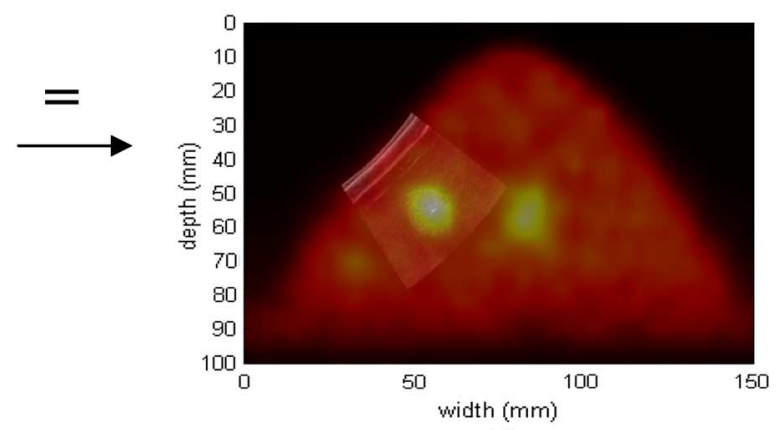

(e)

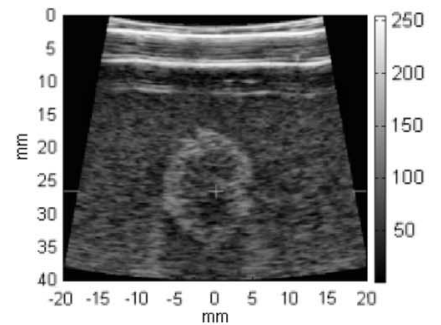

(f)

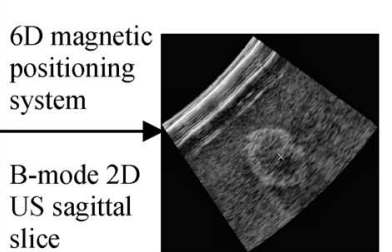

(c)

x $10^{4}$
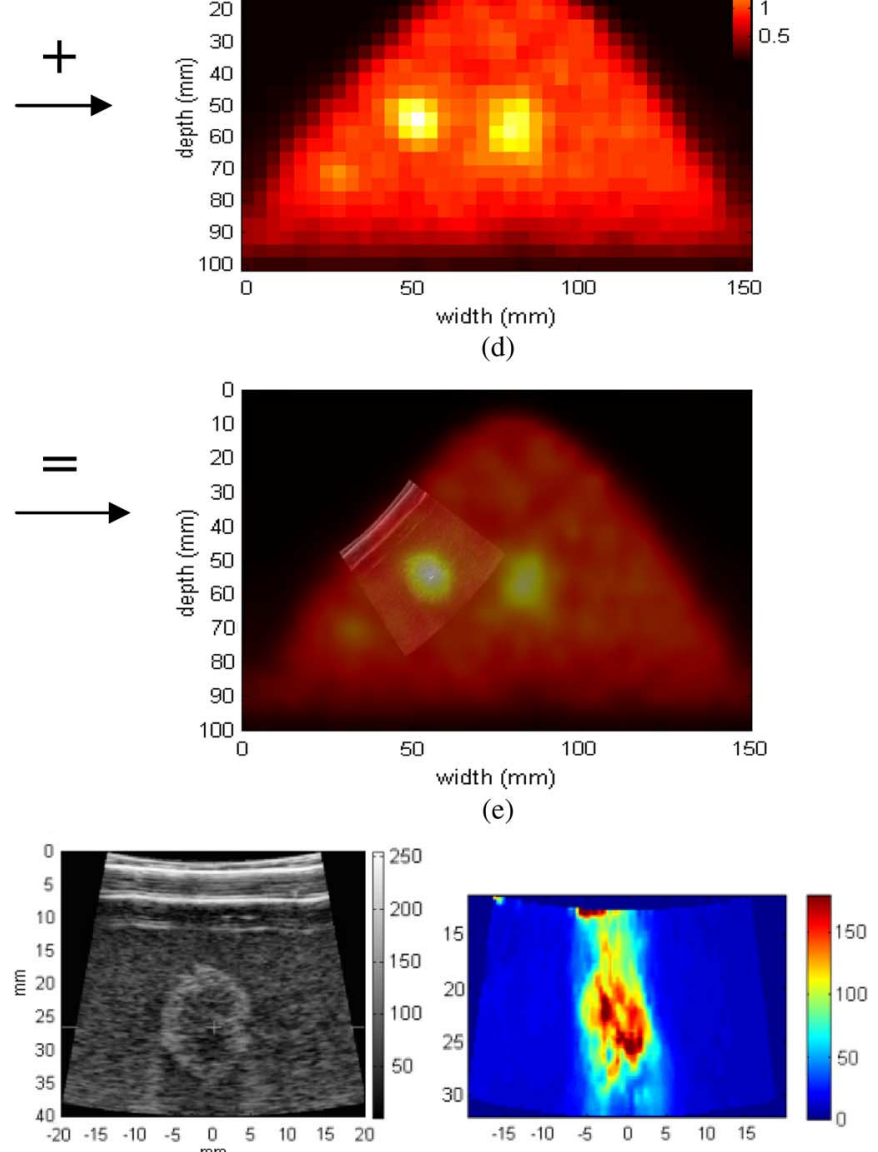

(g)

Fig. 6. PET and US image fusion for the anthropomorphic breast phantom: (a) CT image of the central trans-axial plane in the PET image, (b) recording the US image, (c) B-mode image after rotation by the dip angle, (d) whole-body PET image $(25 \times 38$ pixels $)$, field of view is $100 \mathrm{~mm} \times 150 \mathrm{~mm}$, (e) superimposition of both images (interpolated as $1670 \times 1040$ pixels, field of view $100 \mathrm{~mm} \times$ $150 \mathrm{~mm}),(\mathrm{f})$ and $(\mathrm{g})$ compare B-mode image $(404 \times 452$ pixels, field of view $40 \mathrm{~mm} \times 40 \mathrm{~mm})$ and shear wave elastographic image $(91 \times 124$ pixels, field of view $40 \mathrm{~mm} \times 36 \mathrm{~mm}$ ) of tumour. The dynamic range of B-mode images is $65 \mathrm{~dB}$ and the elasticity range of elastography information is $180 \mathrm{kPa}$.

in the US B-mode image and shows clearly on the elastogram. The small lesion indicated with an arrow in the upper part of Fig. 7(e) and 7(f) is also much more visible in the elastogram. In Fig. 7(e), the outlined arrow points at a dark vertical band. This is due to the shadow of the hard tissue above it. This is

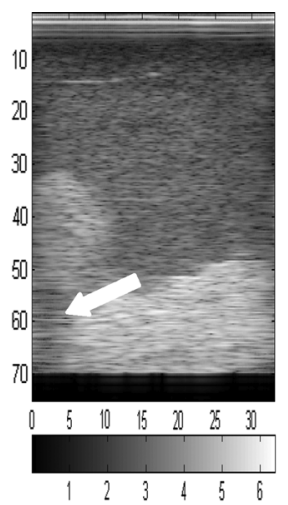

(a) B-mode

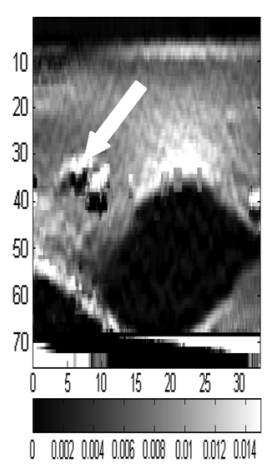

(d) Elastogram

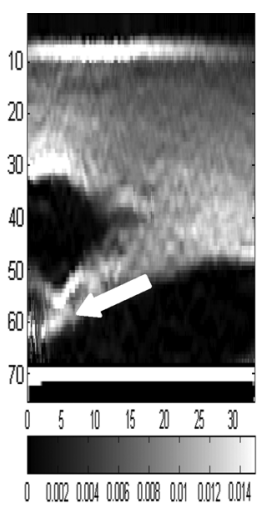

(b) Elastogram

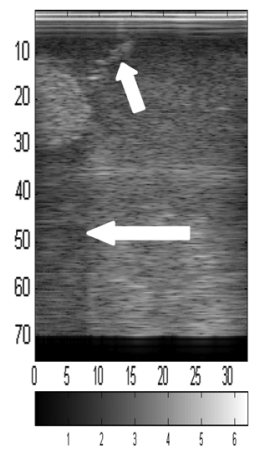

(e) B-mode

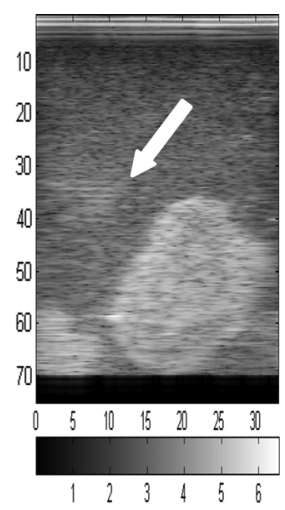

(c) B-mode

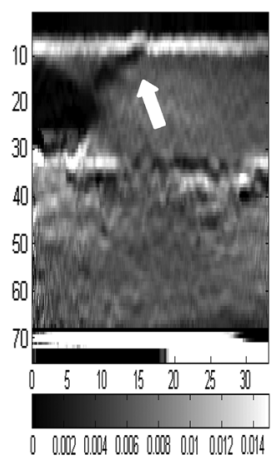

(f) Elastogram
Fig. 7. Comparison of B-mode US image and static elastogram. Young's modulus between tumor and normal tissue mimicking material is 5:1, dynamic range of B-mode images are $65 \mathrm{~dB}$, and dynamic range of strain image is $0-0.015$. All the image fields of view are $33 \mathrm{~mm} \times 75 \mathrm{~mm}$. In (a) and (b), the white arrow shows in static elastography that the strain seems to be higher between two hard lesions, but in fact it is caused by mechanical artifact by former studies [26], [29]. In (c) and (d), the white arrow indicates a small lesion can be easily found by static elastography, while in B-mode image it is hardly to be found. This supports that elastography can be an ideal supplementary tool for conventional B-mode exam, to help clinical biopsy. In (e) and (f), elastography can overtake the shadowing effect compared with B-mode imaging and also it can detect very small-sized lesions, parallel to conventional B-mode imaging. (a) B-mode; (b) Elastogram; (c) B-mode; (d) Elastogram; (e) B-mode; (f) Elastogram.

a well-known artifact of US B-mode imaging, and the effect is absent in the elastogram 7(f).

\section{B. Signa- to-Noise Ratio}

For the PET modality, Fig. 8 shows the signal and background activity of the breast phantom. The signal-to-noise ratio of the background region is 19 . The tumour activity peak value is 2.2 times higher (tumour 1) than the background region (Fig. 8(a)). This is less than the ratio of the activities in tumour and fat medium, and the difference is due to the small size of the tumours compared to the resolution of the scanner, namely partial volume effect. The diameter of the tumour 1 is 1.5 $\mathrm{cm}$. The activity ratio between tumour and fat medium is $4: 1$. According to partial volume effect [30], the measured activity should be around $50 \%$ of the original activity when the PET scanner FWHM spatial resolution is $4 \mathrm{~mm}$. So this explains why we have measured 2.2 of signal to noise ratio for tumour 1. For tumour 2, as the image plane we used did not cross the tumour center but the edge of it, the measured activity is even lower than tumour 1 . For the US modality, the signal-to-noise ratio of the background region is 10 . The signal reflected by 


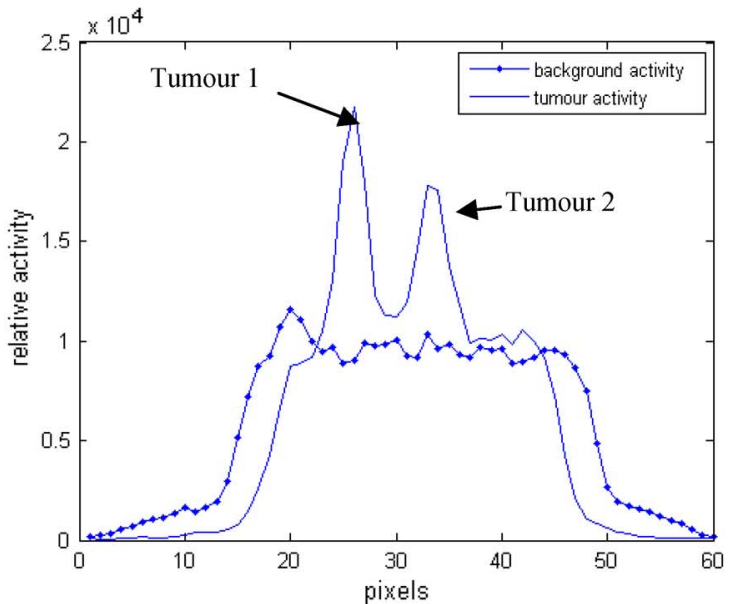

(a)

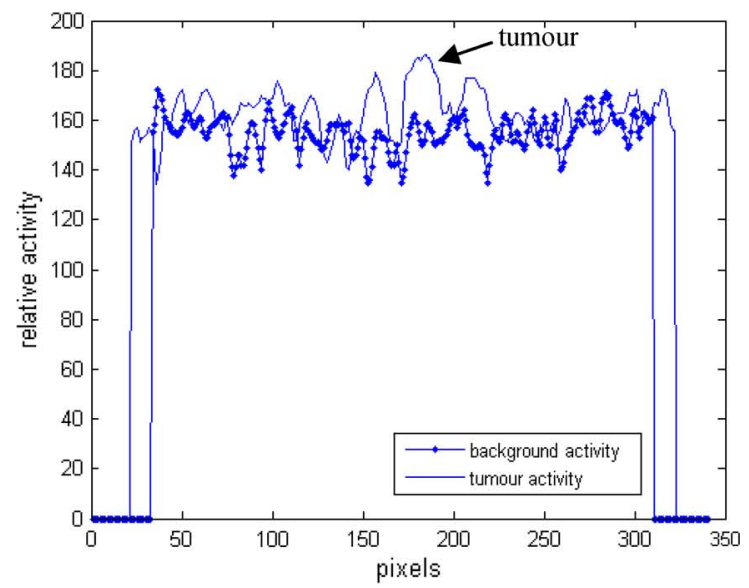

(b)

Fig. 8. (a) Signals of phantom tumour part and background part from PET image. (b) signals of phantom tumour part and background part from SuperSonic B-mode image.

the tumour is only a factor 1.2 larger than the background signal, giving a signal to noise ratio of about 2 . This is a normal situation for US images (Fig. 8(b)).

\section{A Phantom for the ClearPEM/Sonic Project}

Our tests show that it is possible to make phantoms that can be used for testing a multimodal PET/US scanner. If the phantom is prepared with inclusions simulating cancerous lesions with dimensions in the range $1-10 \mathrm{~mm}$, it will be very useful for comparing the performance of the ClearPEM with the performance of standard whole-body PET systems. The Supersonic Aixplorer scanner can detect lesions in the $1 \mathrm{~mm}$ range. The ClearPEM scanner will allow the observation of hot spots in the same size range [31]. The phantom will be an ideal training tool for studying volume registration and image fusion, and for system performance evaluation.

Fig. 9 shows the layout of the ClearPEM scanner and the plastic head contention cone. It also shows how the US probe will be held in position in the combined ClearPEM/Sonic scanner.

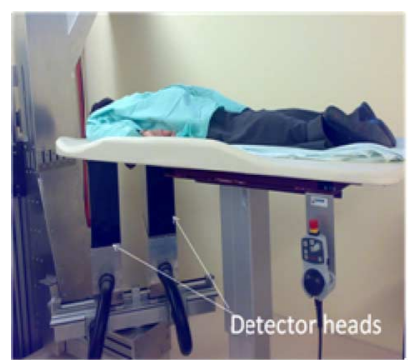

(a)

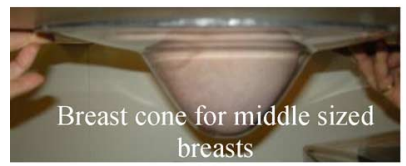

(c)

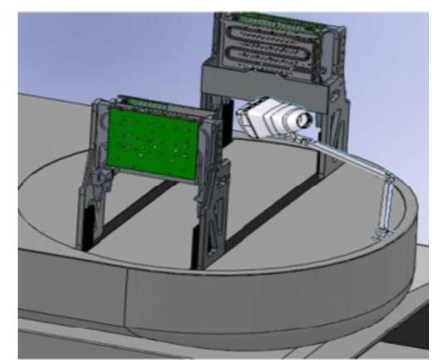

(b)

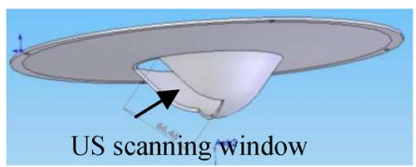

(d)
Fig. 9. ClearPEM/Sonic combination. (a) ClearPEM patient scanner with the patient scanned by a prone position; (b) US probe arm fixed with ClearPEM detector heads; (c) plastic breast contention cone; and (d) US scanning window on the breast contention cone.

\section{Image Registration}

Image registration is an essential step in multimodal imaging. We tested both fiducial marker based image registration and "mutual information" software based image registration for phantom images [32], [33]. Although mutual information based image registration has been reported to be robust for multimodality image registration in case of PET/MR and PET/CT [34], [35], we obtained only very disappointing results. This is probably due to the limited field of view of the US image, and therefore the limited overlap region between the two images, and to the low signal-to-noise ratio [36], particularly for the US image.

For fiducial marker-based registration, on the other hand, we obtained good results. Unfortunately, in the present test, we could not use the radioactive point sources as fiducials for the image superposition because the activity in the fiducials was too low, and we had to rely on the metal screws visible in the CT image. We are nevertheless confident that it is possible to use radioactive point sources for superposing the images. This method can only be used if the breast retention cone immobilizes the breast sufficiently while both images are recorded. Since the fiducial markers are not visible on the US image, we must rely on the 3D magnetic positioning system. In this method, only rigid linear transformations are applied.

\section{E. Conclusion}

We have developed a gelatine-agar-based dual-modality phantom for PET/US breast-imaging research. Gels with acoustic and mechanical properties that closely resemble real tissues such as fat tissue, glandular tissue, fibrous tissue, and carcinoma could be obtained. During the preparation of the phantom, FDG can be added to give the different components activity levels similar to what will be observed during PET scans. Tests with a whole-body PET/CT and US imaging systems confirmed the good properties of the phantom. On the basis of our preliminary tests, we are confident that image registration is possible by using radioactive fiducial markers and a 6D magnetic positioning system. 


\section{ACKNOWLEDGMENT}

The authors thank colleagues from the Laboratory for Instrumentation and Experimental Particle Physics (LIP), Lisbon, especially Catarina M. F. Ortigão and Andreia M. A. Trindade Rodrigues, and the Instituto Português de Oncologia, Porto, for precious help in several phases in this work.

\section{REFERENCES}

[1] World Health Organization International Agency for Research on Cancer, Cancer Incidence and Mortality Worldwide in 2008, Fact Sheets: Breast Cancer [Online]. Available: http://globocan.iarc.fr

[2] World Health Organization, Fact Sheet Number 297, Feb. 2009 [Online]. Available: http://www.who.int/mediacentre/factsheets/fs297/en/ index.html

[3] S. Shapiro, W. Venet, and P. Strax, "Ten- to fourteen-year effect of screening on breast cancer mortality," J. Natural Cancer Inst., vol. 69, pp. 349-355, 1982.

[4] S. A. Feig, G. A. Shaber, and A. Patchefskly, "Analysis of clinically occult and mammographyically occult breast tumors," Amer. J. Roentgenol., vol. 128, pp. 403-408, 1977.

[5] E. L. Thurfjell, L. H. Holmberg, and I. R. Persson, "Screening mammography: Sensitivity and specificity in relation to hormone replacement therapy," Radiology, vol. 203, pp. 339-341, 1997.

[6] R. Kumar, N. Lal, and A. Alavi, "18F-FDG PET in detecting primary breast cancer," J. Nucl. Med., vol. 48, no. 10, p. 1751, 2007.

[7] A. Quon and S. S. Gambhir, "FDG-PET and beyond: Molecular breast cancer imaging," J. Clinical Oncology, vol. 23, no. 8, pp. 1664-1673, 2005.

[8] A. Iagaru, R. Masamed, S. Keesara, and P. S. Conti, "Breast MRI and 18F FDG PET/CT in the management of breast cancer," Ann. Nucl. Med., vol. 21, no. 1, pp. 33-38, 2007.

[9] S. K. Yang, N. Cho, and W. K. Moon, "The role of PET/CT for evaluating breast cancer," Korean J. Radiology, vol. 8, no. 5, pp. 429-437, 2007.

[10] S. J. Siml, J. H. C. L. Hendriks, and S. M. C. Fook-Chong, "Breast ultrasound in women with familiar risk of breast cancer," Ann. Acad. Med., Singapore, vol. 33, no. 5, pp. 600-606, 2004.

[11] N. Houssami, S. Ciatto, L. Irwig, J. M. Simpson, and P. Macaskill, "The comparative sensitivity of mammography and ultrasound in women with breast symptoms: An age-specific analysis," The Breast, vol. 11, no. 2, pp. 125-130, 2002.

[12] G. Rizzatto and R. C. Source, "Breast ultrasound and new technologies," Eur. J. Radiology, vol. 27, pp. 242-249, 1998.

[13] N. Lang, S. Hermann, and S. Hold et al., "Hybrid 3D Sono/PET in a mouse," Eur. J. Nucl. Med. Molecular Imag., vol. 34, pp. 1706-1707, 2007.

[14] M. Tanter, J. Bercoff, A. Athanasiou, and T. Deffieux, "Quantitative assessment of breast lesion viscoelasticity: Initial clinical results using SuperSonic shear imaging," Ultrasound Med. Biol., vol. 34, pp. 1373-1386, 2008

[15] J. S. Huber, Q. Peng, and W. W. Moses, "Multi-modality phantom development," IEEE Trans. Nucl. Sci., vol. 56, no. 5, pp. 2722-2727, Oct. 2009.

[16] J. Varela et al., "Characterization of the clear-PEM breast imaging scanner performance," in Proc. IEEE Nuclear Science Symp. Conf. Rec., 2009, pp. 3487-3490.
[17] P. Lasaygues and M. Pithioux, "Ultrasonic characterization of orthotropic elastic bovine bones," Ultrasonics, vol. 39, no. 8, pp. $567-573,2002$

[18] H. Hasni, F. A. Meah, and A. Norlia, "Ultrasound in the assessment of the palpable breast mass," Ultrasound Assess. Palpable Breast Mass. Med. J. Malaysia, vol. 59, no. 4, pp. 486-494, 2004.

[19] T. A. Krouskop et al., "Elastic moduli of breast and prostate tissues under compression," Ultrason. Imag., vol. 20, pp. 260-274, 1998.

[20] J. Bercoff, M. Tanter, and M. Fink, "Supersonic shear imaging: A new technique for soft tissue elasticity mapping," IEEE Trans. Ultrason. Ferroelectr. Freq. Control, vol. 51, no. 4, pp. 396-409, 2004.

[21] J. Ophir, I. Cespedes, H. Ponnekanti, and Y. Yazdi, "Elastography: A quantitative method for imaging the elasticity of biological tissues," Ultrason. Imag., vol. 13, pp. 186-210, 1991.

[22] T. Varghese and J. Ophir, "Enhancement of echo-signal correlation in elastography using temporal stretching," IEEE Trans. Ultroson. Ferroelectr. Freq. Control, vol. 44, no. 1, pp. 173-179, 1997.

[23] J. Luo, J. Bai, P. He, and K. Ying, "Axial strain calculation using a low-pass digital differentiator in ultrasound elastography," IEEE Trans. Ultronson. Ferroelectr. Freq. Control, vol. 51, no. 9, pp. 1119-1127, 2004.

[24] S. Surti, A. Kuhn, and M. E. Werner et al., "Performance of Philips Genimi TF PET/CT scanner with special consideration for its time-offlight imaging capabilities," J. Nucl. Med., vol. 48, no. 3, pp. 471-480, 2007.

[25] L. M. Popescu, "Iterative image reconstruction using geometrically ordered subsets with list-mode data," in Proc. IEEE Nuclear Science Symp. Medical Imaging Conf., 2004, vol. 6, no. 16-22, pp. 3536-3540.

[26] Y. Cui, J. Li, M. Kadour, and A. Noble, "Displacement and strain estimation of breast tissue using multi-compressed ultrasound RF signals," in Proc. Medical Images and Signals to Clinical Information-InterDisciplinary Research Consortium Plenary 2005, Chancellors, Manchester [Online]. Available: http://www2.wiau.man.ac.uk/miasirc/online-proceedings/papers/22/IRC_MIAS_05_1.pdf

[27] E. L. Madsen, M. A. Hobson, G. R. Frank, and J. B. Weaver, "Anthropomorphic breast phantoms for tesing elastography systems," Ultrasound Med. Biol., vol. 32, no. 6, pp. 857-874, 2006.

[28] D. Zhang, M. Wan, and H. Zhang, "Improvement on the elastic visualization of thermal lesion suing block wavelet shrinkage," in Proc. IEEE Ultrasonics Symp., 2008, no. 2-5, pp. 2017-2020.

[29] J. Li, Y. Cui, R. E. English, and J. A. Noble, "Ultrasound estimation of breast tissue biomechanical properties using a similarity-based nonlinear optimization approach," J. Strain Anal. Eng. Des., vol. 44, no. 5 , pp. 363-374, 2009

[30] M. Soret, S. L. Bacharach, and I. Buvat, "Partial-volume effect in PET tumour imaging," J. Nucl. Med., vol. 48, no. 6, pp. 932-945, 2007.

[31] J. A. Neves et al., "The ClearPEM breast imaging scanner," Nucl. Instrum. Methods Phys. Res. A, 2010, submitted for publication.

[32] W. M. Wells, P. Viola, H. A. S. Nakajimae, and R. Kikinis, "Multimodal volume registration by maximization of mutual information," Med. Image Anal., vol. 1, no. 1, pp. 35-51, 1996.

[33] J. P. W. Pluim, J. B. A. Maintz, and M. A. Viergever, "Mutual information based registration of medical images: A survey," IEEE Trans. Med. Imag., vol. 22, no. 8, pp. 986-1004, 2003.

[34] J. P. W. Pluim, J. B. A. Maintz, and M. A. Viergever, "Image registration by maximization of combined mutual information and gradient information," IEEE Trans. Med. Imag., vol. 19, no. 8, pp. 809-814, 2000.

[35] M. Jenkinson, P. Bannister, M. Brady, and S. Smith, "Improved optimization for the robust and accurate linear registration and motion correction of brain images," NeuroImage, vol. 17, no. 2, pp. 825-841, 2002.

[36] F. Maes, "Multimodality image registration by maximization of mutual information," IEEE Trans. Med. Imag., vol. 16, no. 2, pp. 187-198, 1997. 